Adny Henrique Silva*, Enio Lima Jr, Marcelo Vasquez Mansilla, Roberto Daniel Zysler, Mary Luz Mojica Pisciotti, Claudriana Locatelli, Rajith Kumar Reddy Rajoli, Andrew Owen, Tânia Beatriz Creczynski-Pasa and Marco Siccardi

\title{
A physiologically based pharmacokinetic model to predict the superparamagnetic iron oxide nanoparticles (SPIONs) accumulation in vivo
}

DOI 10.1515/ejnm-2017-0001

Received January 17, 2017; accepted March 10, 2017; previously published online March 30, 2017

Abstract: Superparamagnetic iron oxide nanoparticles (SPIONs) have been identified as a promising material for biomedical applications. These include as contrast agents for medical imaging, drug delivery and/or cancer cell treatment. The nanotoxicological profile of SPIONs has been investigated in different studies and the distribution of SPIONs in the human body has not been fully characterized. The aim of this study was to develop a physiologically-based pharmacokinetic (PBPK) model to predict the pharmacokinetics of SPIONs. The distribution and accumulation of SPIONs in organs were simulated taking into consideration their penetration through capillary walls and their active uptake by specialized macrophages in the liver, spleen and lungs. To estimate the kinetics of SPION uptake, a novel experimental approach using primary macrophages was developed. The murine PBPK model was validated against in vivo pharmacokinetic data, and accurately described accumulation in liver, spleen and lungs. After validation of the murine model, a similar PBPK approach was developed to simulate the distribution of SPIONs in humans. These data demonstrate the

\footnotetext{
*Corresponding author: Adny Henrique Silva, Departamento de Ciências Farmacêuticas, Universidade Federal de Santa Catarina, P.O. Box 476, Florianópolis, SC, 88040-900, Brazil, Phone: +55483721 2212, Fax: +55 4837219542 , E-mail:adnyh@yahoo.com.br; adnyhe@gmail.com Enio Lima Jr, Marcelo Vasquez Mansilla, Roberto Daniel Zysler and Mary Luz Mojica Pisciotti: División of Resonancias Magnéticas, Centro Atómico Bariloche/CONICET, S. C. Bariloche 8400, Argentina Claudriana Locatelli: Curso de Farmácia, Universidade do Oeste de Santa Catarina, Videira, SC, 89560-000, Brazil; and Universidade do Alto Vale do Rio do Peixe, Caçador, SC, 89500-000, Brazil Rajith Kumar Reddy Rajoli, Andrew Owen and Marco Siccardi: Molecular and Clinical Pharmacology, Institute of Translational Medicine, University of Liverpool, Liverpool, UK Tânia Beatriz Creczynski-Pasa: Departamento de Ciências Farmacêuticas, Universidade Federal de Santa Catarina, P.O. Box 476, Florianópolis, SC, 88040-900, Brazil
}

utility of PBPK modeling for estimating biodistribution of inorganic nanoparticles and represents an initial platform to provide computational prediction of nanoparticle pharmacokinetics.

Keywords: accumulation; biodistribution; nanoparticles; physiologically based pharmacokinetic model; prediction.

\section{Introduction}

Superparamagnetic iron oxide nanoparticles (SPIONs) are a nanotechnology with favorable characteristics for biological and medical applications such as magnetic resonance imaging (MRI) (1, 2), drug delivery (2), cellular therapy, cell labeling $(3,4)$, tissue repair (5) and cancer treatment through hyperthermia (6). However, the biomedical applications of SPIONs may be limited by their low biocompatibility and toxicity. The nanotoxicological profile of SPIONs has been investigated in different studies, highlighting the generation of oxidative stress, cell proliferation inhibition, mitochondrial damage and endoplasmic reticulum stress (7-10). Knowledge of the in vivo biodistribution of SPIONs is essential for a robust assessment of all medical applications. Although the toxicology of SPIONs has been investigated, there is a current paucity of data relating to in vivo pharmacokinetics. Thorough understanding of the mechanisms regulating nanoparticles (NPs) pharmacokinetics is essential to design effective and safe NPs for biomedical applications.

Physiologically based pharmacokinetic (PBPK) modeling combines mathematical equations to describe the anatomical, physiological and molecular processes regulating pharmacokinetics, with in vitro data to simulate and predict the absorption, distribution, metabolism and elimination (ADME) of conventional and nano-enabled medicines $(11,12)$. Several processes influence the distribution of NPs in the human body and the ADME of NPs can substantially differ from 
traditional formulations, complicating the development of PBPK models. Macrophages are specialized cells that can phagocytose nanomaterials and represent an essential component of the reticuloendothelial (RE) system in organs such as the liver and spleen $(11,13,14)$. Moreover, the "enhanced permeability and retention" (EPR) effect increased uptake of NPs in tumors and inflamed sites, and represents a passive mechanism for NP delivery of anticancer drugs (15).

The first applications of PBPK for simulation of NPs distribution emerged in 2006 as a potentially valuable tool to inform about NP hazard, as recently indicated in the Organization for Economic Cooperation and Development (OECD) guidelines (11). One of the main challenges in the development of PBPK modeling for NPs is accurate estimation of the correlation between NPs properties and distribution patterns in vivo. Experimental in vitro approaches have recently been developed to describe the interaction between NPs and different cell populations, providing methods to better understanding distribution of NPs. Importantly, such data can be integrated into the PBPK modeling (12). Size (magnetic and hydrodynamic diameters), shape, superficial charge and chemical composition are some properties of SPIONs that can determine their uptake by macrophages, accumulation in the cells, penetration through capillaries as well as kidney ultrafiltration $(15,16)$.

The main aim of this study was to create a PBPK model able to predict the pharmacokinetics of nanostructured systems in mice and humans. The model was used to gain a better understanding of the nanotoxicological potential of SPIONs with neutral mPEG2000 coating. Additionally, a novel experimental approach was developed to estimate the kinetics of NPs uptake into macrophages, with subsequent integration into the PBPK model.

\section{Materials and methods}

\section{Materials}

The cell culture medium (RPMI and IMDM), fetal bovine serum (FBS), Hank's balanced salt solution (HBBS), ficoll paque, EDTA and reagents used in the ferrozine assay were purchased from Sigma-Aldrich (Munich, Germany). The DMEM medium was purchased from Cultilab (São Paulo, Brazil) and antibiotics and HEPES were purchased from GIBCO (Grand Island, NY, USA). The MicroBeads, macrophage-colony stimulating factor (M-CFS) and columns used in the separation and differentiation of CD14+ monocytes to macrophages were purchased from Miltenyi Biotec Inc (Auburn, AL, USA). The blood buffy coats were obtained from regional blood transfusion center (Liverpool, $\mathrm{UK})$.

\section{Superparamagnetic iron oxide nanoparticles (SPIONs)}

SPIONs were provided by CNEA - Atomic Center of Bariloche, Bariloche, Argentina and are composed of superparamagnetic iron oxide $\left(\mathrm{Fe}_{3} \mathrm{O}_{4}\right) \mathrm{NP}$, coated with oleic acid and the conjugate phosphatidyletanolamine - methoxy polyethylene glycol with $m w 2000$ (PE-mPEG2000), as illustrated in Figure S1 (Supplementary material). The NPs were prepared by thermal decomposition of organometallic precursor iron (III) acetylacetonate (Aldrich, St. Louis, MO, USA) at high temperature $(17,18)$. NPs were coated with a bilayer composed of oleic acid (Sigma-Aldrich, St. Louis, MO, USA) and PE-mPEG2000(1,2-dioleoyl-sn-glycero-3-phosphoethanolamine-N-[methoxy(polyethylene glycol)-2000], ammonium salt, 18:1 PE-PEG2000, purity $>99 \%$, Avanti Lipids, Alabaster, AL, USA). The ratio between nanoparticle to conjugate was 1:2 wt (1 mole of nanoparticle to 0.16 of mPEG phospholipid conjugate). Morphological and structural characterization of the SPIONs was made by transmission electron microscopy (TEM). Size and surface charge were determined by dynamic light scattering and laser-Doppler anemometry, using a Zetasizer Nano ZS (Malvern Instruments, Worcestershire, UK), equipped with $173^{\circ}$ scattering angle, as previously described (19). In addition, Fourier transform infrared spectroscopy was performed to evaluate the formation of the organic bi-layer. Magnetic characterization of the SPIONs was performed by magnetization curves as a function of an applied field at room temperature (in a vibrating sample magnetometer, VSM, Lakeshore) and temperature (SQUID magnetometer, MPMS6 - Quantum Designs).

\section{Animals and treatments}

We maintained 6-8 week-old Swiss albino male mice in accordance with the Ethics Committee for Animal Use as approved by the Animal Care and Use Committee (Opinion number 01/2012). The mice were housed at $23 \pm 2^{\circ} \mathrm{C}$ and a relative humidity of $50-60 \%$ under a $12: 12 \mathrm{~h}$ light: dark cycle with food and water given ad libitum. The weighed animals were divided into four groups of 10 mice each. As there are currently no guidelines or standard methodologies for in vivo toxicity, we chose to estimate doses and treatment/monitoring time according to the Organization for Economic Co-operation and Development (OECD) guidelines 420 for investigating the oral toxicity of any new substance. The control group received only vehicle (saline) and the treated groups received 510, 1020, $2040 \mu \mathrm{g} /$ mouse of SPIONs. The solutions were administered intravenously in a single dose. On the 14th day after the treatment, the animals were sacrificed following standard procedure and the liver, spleen, lung, kidney, heart and brain were harvested for SPION quantification.

SPIONs quantification: The samples of each organ were processed through dehydration and maintained frozen until the moment of analysis. Specifically, liver, lung, heart, brain, spleen and kidney were extracted and fixed in formalin solution for $48 \mathrm{~h}$ and then through a series of ethanol solutions 70,96 , and $100 \% \mathrm{v} / \mathrm{v}$ successively. Subsequently, the SPIONs were quantified ex vivo, i.e. in the animal tissues by means of magnetization measurements, using a calibration curve and the superparamagnetic property of the system. This was conducted as previously described and SPIONs are completely distinct from the endogenous iron (20). Briefly, the magnetization curve as a function of the applied field for a known amount of 
tissue was measured in VSM and SQUID (very low magnetic signal). The diamagnetic component of the curve was obtained from the linear fit of the high field region ( $>7 \mathrm{kOe}$ ). The saturation magnetization of the magnetic (or superparamagnetic) component of the curve was obtained from the linear coefficient of the linear fit. By comparing this value with the saturation magnetization of the NPs measured previously, it was possible to determine the NPs concentration within the sample. Four values were obtained for each magnetization curve (from the four quadrants), and the arithmetic mean of these four values was used. This technique allows measurement up to $1 \mu \mathrm{g}$ of SPIONs.

\section{Experimental ex vivo/in vitro assays}

Cell culture: Murine macrophage cell line (J774) was obtained from Banco de Células do Rio de Janeiro (BCRJ, Brazil). The cells were cultured in DMEM medium supplemented with 10\% heat-inactivated fetal bovine serum, $100 \mathrm{U} / \mathrm{mL}$ penicillin, $100 \mu \mathrm{g} / \mathrm{mL}$ streptomycin, $10 \mathrm{mM}$ HEPES and maintained at $37^{\circ} \mathrm{C}$ in a $5 \% \mathrm{CO}_{2}$ humidified atmosphere. Every 2 days, the cells were passaged, and the medium was replaced.

Human PBMCs (peripheral blood mononuclear cells) were isolated from buffy coats by gradient density centrifugation. An aliquot of buffy coats were slowly added to a Ficoll-paque reagent and centrifuged (30 min, $400 \times \mathrm{g}$ ). PBMCs were removed from the solution, washed with HBSS and centrifuged ( $5 \mathrm{~min}, 400 \times \mathrm{g}$ ). The cell pellet was resuspended and cultured in RPMI 1640 media containing 10\% FBS. After $24 \mathrm{~h}$ of incubation, CD14+ monocytes were isolated for generation of macrophages. For that, CD14 MicroBeads Kit (for human samples), from Miltenyi Biotec Inc., was used according to the manufacturer's instructions.

Initially, $10^{7}$ cells were washed with HBBS and resuspended in a buffer (HBBS, 0.5\% of albumin, 2 mM EDTA) and CD14 MicroBeads and refrigerated $\left(2-8^{\circ} \mathrm{C}\right)$ for $15 \mathrm{~min}$. After incubation, cells were washed with buffer and centrifuged for $10 \mathrm{~min}$ at $300 \times \mathrm{g}$. The supernatant fraction was aspirated completely and cells were resuspended in buffer. The CD14+ separation was performed using magnetic MACS separation applying the labeled cells into the magnetic columns. The CD14+ cells were eluted and counted and platted using IMDM medium supplement with $20 \%$ FBS and $100 \mathrm{ng} / \mathrm{mL}$ macrophage-colony stimulating factor (M-CFS). Cells were maintained at $37^{\circ} \mathrm{C}$ in a $5 \%$ $\mathrm{CO}_{2}$ humidified atmosphere, the medium was replaced every 2 days and after 7 days macrophages were used to determine SPION uptake.

Quantitative human macrophage uptake of SPIONs: Primary macrophages $\left(2 \times 10^{6}\right)$ were incubated with crescent concentrations $(1,10$ and $100 \mu \mathrm{g} / \mathrm{mL})$ of SPIONs for 1,6 and $24 \mathrm{~h}$. After the incubation time, the uptake of SPIONs was determined using colorimetric ferrozine assay according to Riemer and collaborators (21) with some modifications. The cells were washed tree times using the buffer HBBS to remove all the SPIONs from the supernatant fraction and were detached using trypsin. The number of cells in each well was counted to determine the SPIONs uptake per cell. After centrifugation for $5 \mathrm{~min}$ at $400 \times \mathrm{g}$ cells were lysed with $\mathrm{NaOH}$ for $2 \mathrm{~h}$ on a shaker in a humidified atmosphere. Aliquots of cell lysates were used to quantify the iron content adding the iron-releasing reagent (freshly mixed solution of $1.4 \mathrm{M} \mathrm{HCl}$ and $4.5 \% \mathrm{w} / \mathrm{v}$ of $\mathrm{KMnO}_{4}$ in water) and $\mathrm{HCl} 10 \mathrm{mM}$. These mixtures were incubated overnight at $60^{\circ} \mathrm{C}$ in a fume hood. Once the samples reached the room temperature, the iron-detection reagent (6.6 mM ferrozine, $6.5 \mathrm{mM}$ neocuproine, $2.5 \mathrm{M}$ ammonium acetate, $1 \mathrm{M}$ ascorbic acid dissolved in water) was added and after $30 \mathrm{~min}$ of incubation an aliquot of each sample were transferred to a 96 well plate and read in a microplate reader at $560 \mathrm{~nm}$. The iron content of samples was determined comparing the results with the calibration curve constructed in parallel $\left(\mathrm{FeCl}_{3}\right.$ standards, $\mathrm{NaOH} 50 \mathrm{mM}$, iron-releasing reagent and iron-detection reagent). The macrophage uptake was determined using a standard calibration curve. The results were expressed as concentration of iron/cell, based on the number of cells in each well.

Quantitative murine macrophage uptake of SPIONs: For mice a cell line was used for the SPIONS uptake evaluation since the blood volume required to isolate a sufficient number of monocytes would be extremely high. Murine macrophages cell line $\left(1 \times 10^{6}\right)$ was incubated with crescent concentrations $(1,10$ and $100 \mu \mathrm{g} / \mathrm{mL})$ of SPIONs for 1,6 and $24 \mathrm{~h}$. After the incubation time, the uptake of SPIONs was determined using colorimetric ferrozine assay as previously described. The macrophage uptake was determined using a standard calibration curve. The results were expressed as concentration of iron/cell, based on the number of cells in each well.

\section{Physiologically-based pharmacokinetic (PBPK) model}

The structure of the PBPK model is summarized in Figure 1. The PBPK model is a mathematical representation of the molecular, physiological and anatomical processes defining the distribution of SPIONs. The organ weights of mice are presented in Table S1 (Supplementary material) and were calculated as a percentage of the total body weight, according to previous works (22-24) and experimental results. The regional blood flows for mice were defined as previously described $(23,25-27)$ and are summarized in the Supplementary material (Table S2). Human demographics and anatomical factors such as height, weight, age, gender and body mass index (BMI) were defined as previously described (28). These values were used to simulate organ and tissue volume (liver, lung, kidney, heart, brain, spleen, adipose, intestine, muscles, testes and skin) through allometric equations.

All organs were connected with consideration of regional blood flow and NPs excretion was simulated through the biliary and urinary routes as previously described (29). The urinary excretion constant is described in the Supplementary Table S3 and was optimized for mice to best agree with the in vivo data (30). A factor was included in the model to calculate the biliary excretion, with variability ranging from $70 \pm 20 \%$. For the human PBPK model the urinary excretion constant as well as the biliary excretion equation used for the mice model was applied. The urinary excretion was defined as follow:

$$
\text { Urinary_excretion }=\mathrm{k}_{\text {urinary excretion }} * \mathrm{~B}_{\mathrm{NPs}}
$$

where $\mathrm{k}_{\text {urinary excetion }}\left(\mathrm{min}^{-1}\right)$ is the urinary constant and $\mathrm{B}_{\mathrm{NPs}}(\mu \mathrm{g} / \mathrm{mL})$ is the concentration of NPs in the blood.

The distribution and accumulation of NPs in organs was simulated taking into account the passive diffusion of NPs through the capillary endothelium and the active uptake of NPs by specialized macrophages in the liver, spleen and lung (30). The capillary endothelium characteristics of each organ were described and the influence of the capillary fenestrations on the NPs movement was 


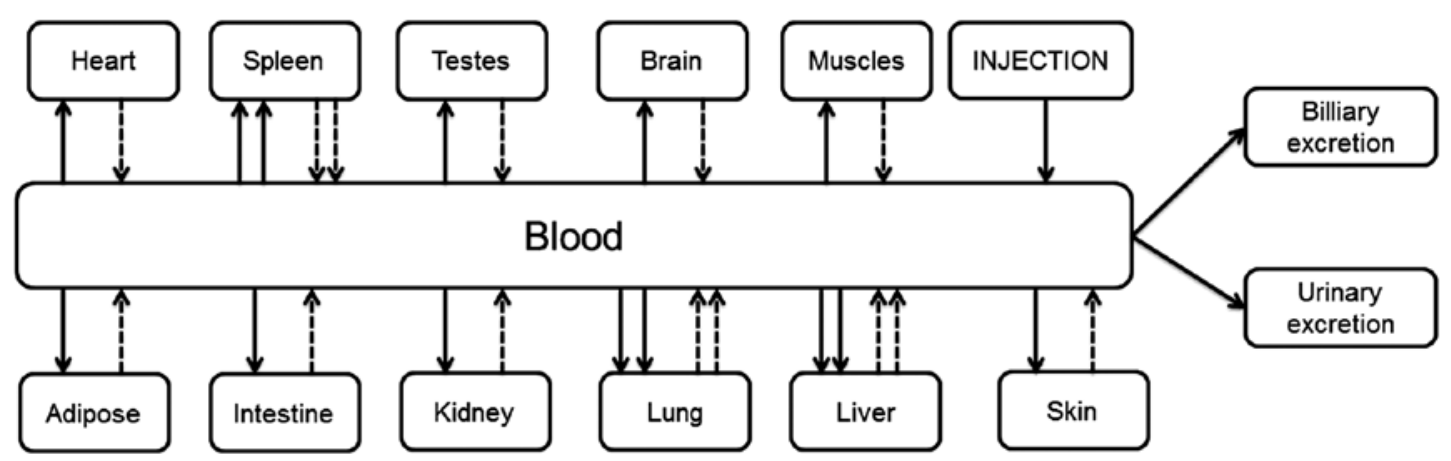

Figure 1: Schematic diagram of the murine and human PBPK models. The single black arrow indicates the passive diffusion of NPs through the capillary fenestrations; the double black arrows represent the passive diffusion plus the active uptake of NPs by macrophages. The dashed arrows indicate the release of NPs from the organ or tissues to the systemic circulation. The diagram also shows the biliary and urinary excretion of NPs.

mathematically represented in the PBPK model (30). The description of the capillary endothelium and the penetration constants included in the model are described in the Supplementary Table S4. The capillary organ uptake was defined by the following equation as previously described (30):

$$
\mathrm{k}_{\text {organNPs }}=\mathrm{B}_{\mathrm{CT}} *\left(\mathrm{Q}_{\text {organ }} / \mathrm{V}_{\text {blood }}\right)
$$

where $\mathrm{k}_{\text {organNPs }}\left(\mathrm{min}^{-1}\right)$ is the constant of penetration through the capillary endothelium, $\mathrm{B}_{\mathrm{CT}}$ is a factor describing the capillary fenestration, $\mathrm{Q}_{\text {organ }}(\mathrm{mL} / \mathrm{min})$ represents the regional blood organ flow and $\mathrm{V}_{\text {blood }}$ $(\mathrm{mL})$ is the total blood volume.

Tissue-resident macrophages are present mainly in the liver, spleen and lung and have as one of their functions the capacity to clear pathogens, cell debris and particles, mediating the uptake and distribution of NPs $(31,32)$. Uptake of SPIONS by murine macrophage cell line and primary human monocyte-derived macrophages were quantified at different time points using a colorimetric assay as described above. The macrophage intrinsic clearance for liver, spleen and lung was derived considering the number of macrophages in each organ using the following equation:

$$
\mathrm{CL}_{\text {int, organ }}=\mathrm{CL}_{\text {int }} * \mathrm{~N}_{\text {macrophages }} * \mathrm{~W}_{\text {organ }}
$$

where $\mathrm{CL}_{\text {int, organ }}(\mathrm{mL} / \mathrm{min}$ /organ) is the intrinsic clearance for each organ, $\mathrm{CL}_{\text {int }}$ is the clearance measured in vitro $(\mathrm{mL} / \mathrm{min} / \mathrm{million}$ of murine macrophage cell line for mice model and primary human monocyte-derived macrophages for human one), $\mathrm{N}_{\text {macrophages }}$ is the number of macrophages per gram of tissue: $8.6 \times 10^{5}$ for human liver (33), $1 \times 10^{7}$ for mouse liver (34), $2 \times 10^{6}$ for human spleen (35), $4.5 \times 10^{6}$ for mouse spleen (36), $1.1 \times 10^{6}$ for human lung (23), and $3 \times 10^{7}$ for mouse lung (37). $\mathrm{W}_{\text {organ }}(\mathrm{g})$ is the weight of the organ in grams. Additionally, we have included a factor in the model where only a variable percentage of cells in the liver, spleen and lung can mediate uptake of SPIONs $(70 \pm 20 \%)$. The uptake of SPIONs is further regulated by the flow of blood to the organs. Consequently the total NPs uptake by macrophages was described by the following equation to derive the organ clearance from blood for each organ:

$$
\mathrm{CL}_{\text {organ }}=\left(\mathrm{Q}_{\text {organ }} * \mathrm{CL}_{\text {int }}\right) /\left(\mathrm{Q}_{\text {organ }}+\mathrm{CL}_{\text {int }}\right)
$$

where $\mathrm{CL}_{\text {organ }}$ is organ clearance from blood, $\mathrm{Q}_{\text {organ }}(\mathrm{mL} / \mathrm{min})$ is the regional organ blood flow and $\mathrm{CL}_{\text {int }}\left(\mathrm{mL} / \mathrm{min} / 1 \times 10^{6}\right.$ cell) represents the intrinsic clearance for each organ.
The release rate of NPs from macrophages and capillaries for the mouse model were optimized to best agree with the accumulation described in mice. For the human model we used the same constants validated for the mice model. A detailed description of constants is described in the Supplementary material. Equations used to define the release of SPIONs from macrophages and capillaries are defined as follow:

$$
\begin{aligned}
& \text { Organ_release_cappilary }=\mathrm{k}_{\text {organ_release_cap }} * \mathrm{~N}_{\text {organ.NPs }} \\
& \text { Organ_release_macrophage }=\mathrm{k}_{\text {organ_release_macrophage }} * \mathrm{~N}_{\text {organ.NPs }}
\end{aligned}
$$

where $\mathrm{k}_{\text {organ_release_cap }}\left(\mathrm{min}^{-1}\right)$ and $\mathrm{k}_{\text {organ_release_macrophage }}\left(\mathrm{min}^{-1}\right)$ represent the constant defining the NP release from capillary and macrophages; $\mathrm{N}_{\text {organ.NPs }}$ means the amount of NPs presented in the organ. The model was implemented in Matlab version 2014a using Simbiology toolbox.

\section{Statistical analysis}

The results of in vitro experiments were presented as means \pm standard deviation (SD) of nine wells derived from three independent cultures and compared by two-way ANOVA, followed by Bonferroni's test. The results of in vivo studies were presented as mean \pm SEM $(\mathrm{n}=7)$ and data was compared by one-way ANOVA, followed by Dunnett's test. ${ }^{\star} \mathrm{p}<0.05$ was taken as statistically significant.

\section{Results}

\section{SPIONs characterization}

The SPIONs evaluated in this paper had spherical shape as demonstrated by TEM in the Figure S2/A. The $\mathrm{Fe}_{3} \mathrm{O}_{4}$ particles presented high crystallinity and mean diameter of $21 \mathrm{~nm}(\sigma=0.10)$. The hydrodynamic diameter obtained for SPIONs using DLS (dynamic light scattering) was $168.9 \pm 1.1 \mathrm{~nm}$ and is presented in the Figure S2/B. The SPIONs exhibited a negative surface charge of 
$-18.2 \pm 5.18 \mathrm{mV}$ (data not shown). The formation of the bilayer leads to an increase in the hydrodynamic diameter of the system which results in the difference of size from TEM to DLS analysis. In addition, the FT-IR spectrum of the SPIONs coated with mPEG2000 magnetically separated clearly evidences the presence of PE-mPEG2000 complex and oleic acid, confirming the formation of the bi-layer. Magnetization curves as a function of applied field $(\mathrm{MxH})$ and temperature (MxT) are presented in the Figure S2/C and S2/D, respectively. $\mathrm{MxH}$ curve at $300 \mathrm{~K}$ indicates a saturation magnetization of $M_{s}=72 \mathrm{emu} / \mathrm{g}$ and the curve presents non-hysteresis as expected for superparamagnetic behavior. Moreover, the MxT curve confirms the superparamagnetic behavior of the samples at room temperature with mean blocking temperature around $170 \mathrm{~K}$ (see inset of Figure S2/D), in addition, it clearly shows the Verwey transition at $\mathrm{T}_{\mathrm{V}} \sim 110 \mathrm{~K}$, confirming the formation of $\mathrm{Fe}_{3} \mathrm{O}_{4}$.

\section{Tissue distribution in vivo}

Following intravenous administration, the SPIONs were detectable in the spleen, liver, heart, lung, brain and kidney. The SPIONs could be quantified in all organs with the exception of the kidney of mice treated with the medium dose. The concentration of SPIONs in each organ is summarized in Figure 2. The concentration accumulated in organs was determined through the intrinsic magnetism of SPIONs. A significant accumulation of SPIONs was observed in the liver, spleen, heart and lung of the mice treated with $510 \mu \mathrm{g}$ of SPIONs. For the group of animals that received a dose of $1020 \mu \mathrm{g}$, a higher NP accumulation was seen in the spleen, liver and lung. Considering the

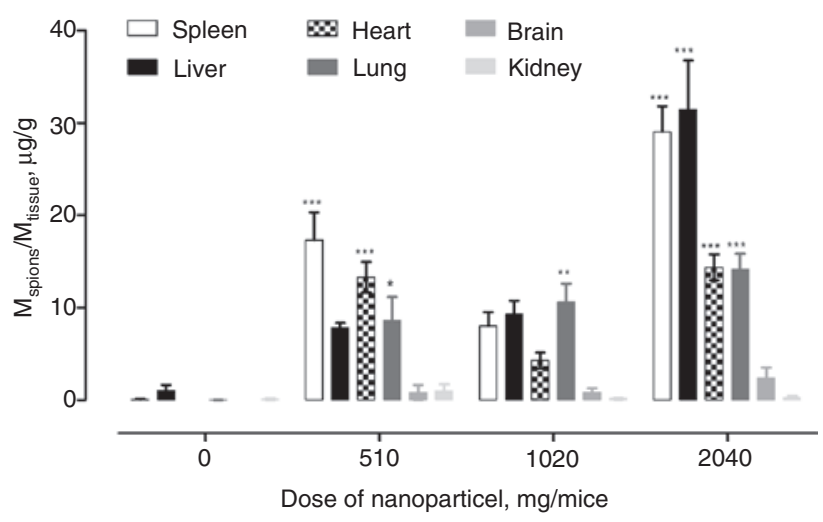

Figure 2: SPIONs accumulation in mice 14 days after the administration. The amount of SPIONs accumulated in organs was determined by magnetization. ${ }^{*} p \leq 0.05,{ }^{* \star} p \leq 0.01$ and ${ }^{* \star *} p<0.001$ using ANOVA followed by Dunnet's test when compared with the control group. mice treated with $2040 \mu \mathrm{g}$, the highest SPIONs accumulation was detected in the liver and spleen.

\section{Murine macrophage uptake of SPIONs and macrophage clearance}

Figure 3A shows the uptake profile of SPIONs by the murine macrophages after 1,6 and $24 \mathrm{~h}$ of incubation. For mice macrophage the uptake of SPIONs was concentration-dependent, with the highest uptake of SPIONs at $100 \mu \mathrm{g} / \mathrm{mL}$ for all the time point evaluated. Incubation for $1 \mathrm{~h}$ resulted in an increase in SPION uptake compared to the 6 and $24 \mathrm{~h}$ incubations. The macrophage clearance was determined using the equation described in Materials and methods and data obtained with the mean of the three concentrations evaluated in $6 \mathrm{~h}$ of treatment was included in the mice model.

\section{Primary human macrophage uptake of SPIONs and macrophage clearance}

Figure 3B shows the uptake profile of SPIONs by the macrophages after 1,6 and $24 \mathrm{~h}$ of incubation. The results suggest that human macrophage uptake of SPIONs is timeand concentration-dependent, with the highest uptake of SPIONs at $100 \mu \mathrm{g} / \mathrm{mL}$. Incubation for $24 \mathrm{~h}$ resulted in an increase in SPION uptake compared to the 1 and $6 \mathrm{~h}$ incubations. The macrophage clearance was determined using the equation described in Materials and methods and the data obtained with the mean of the three concentrations evaluated in $6 \mathrm{~h}$ of treatment was included in the human model.

\section{Physiologically-based pharmacokinetic (PBPK) model}

\section{Murine PBPK modeling}

The PBPK model generated was compared against in vivo results and showed to be effective in the prediction of the SPION distribution. The in vivo data are summarized in Figure 4. The simulated SPIONs distribution was in good agreement with the in vivo accumulation, with maximum difference of three-fold for the brain. The accumulation of SPIONs in the heart was higher in vivo compared to the PBPK prediction for the $510 \mu \mathrm{g}$ dose. The simulated SPIONs concentrations were $1.32 \mu \mathrm{g} / \mathrm{g}$ of tissue vs. $13.3 \mu \mathrm{g} / \mathrm{g}$ in vivo. For the kidney, the accumulation of SPIONs was higher in 

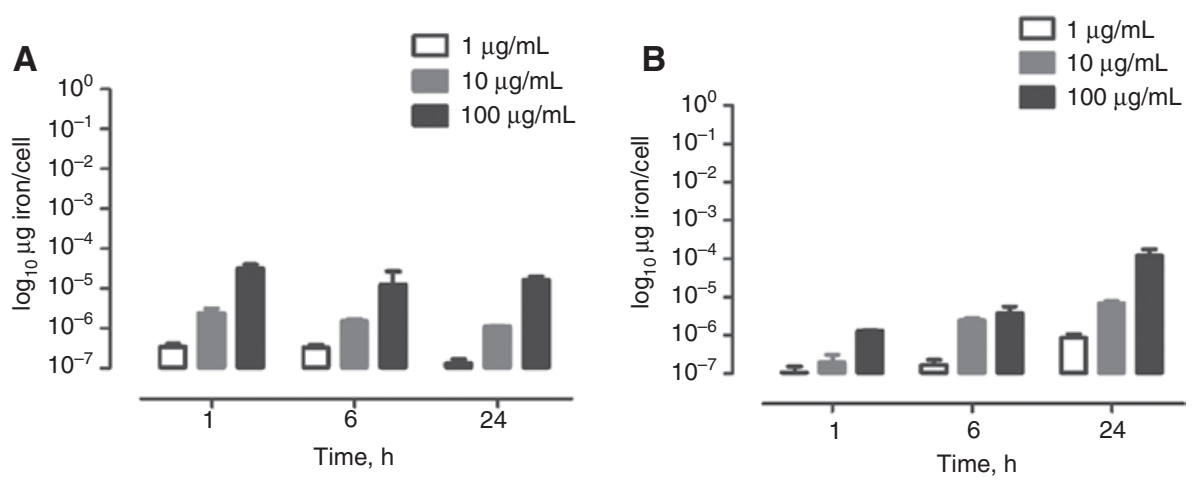

Figure 3: Murine macrophage (A) and primary human macrophage uptake (B) of SPIONs after incubation with 1,10 and $100 \mu \mathrm{g} / \mathrm{mL}$ of NPs for 1,6 and $24 \mathrm{~h}$. The data represent means \pm SD of nine wells derived from three independent culture of murine macrophage cell line and monocyte-macrophage derived.

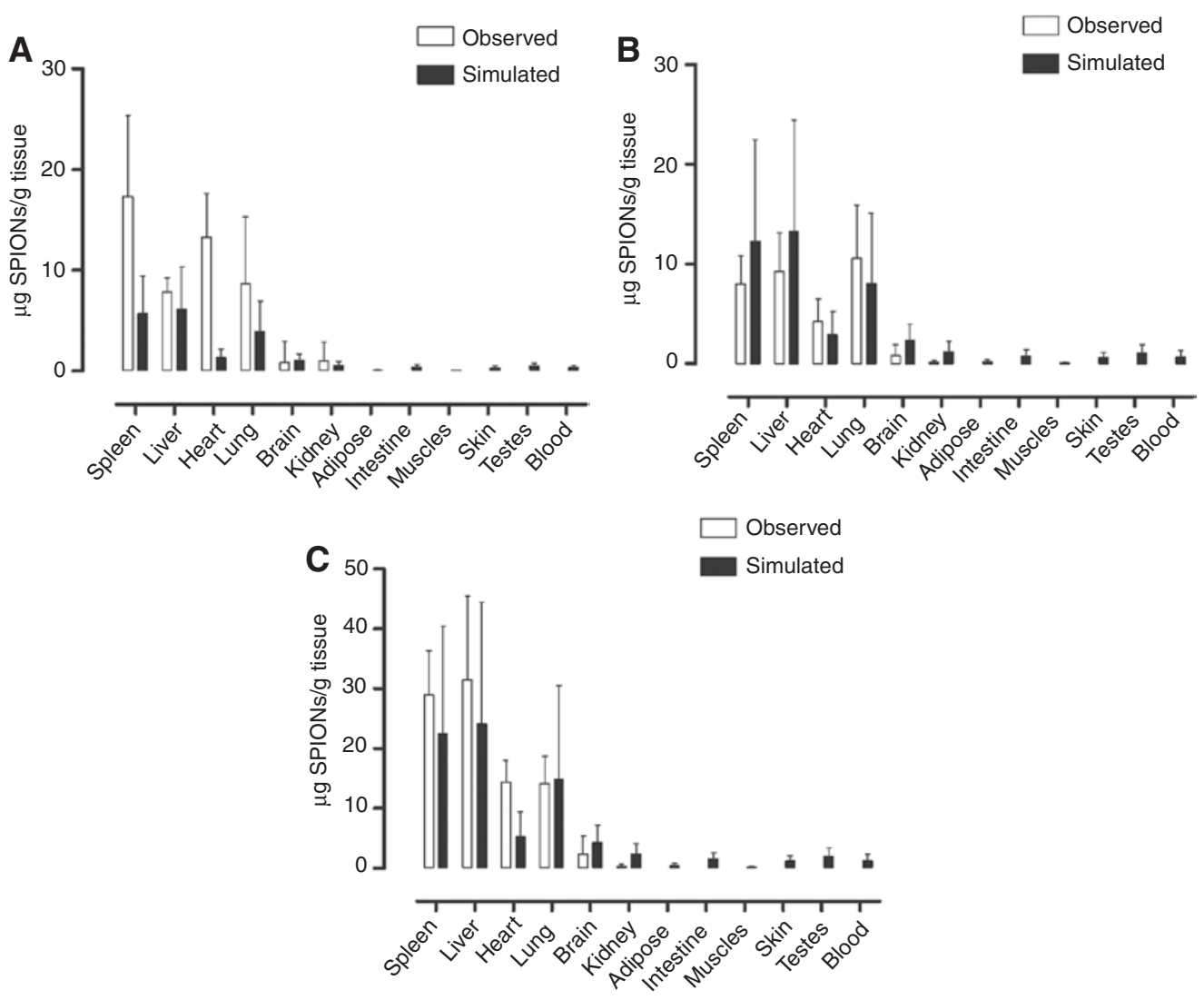

Figure 4: Simulated and in vivo data accumulation of SPIONs in mice. The dose simulated and administered in mice was (A) 510, (B) 1020 and (C) $2040 \mu \mathrm{g} / \mathrm{mouse}$ and the results are expressed as microgram of SPIONs per gram of tissue. The equivalent concentration of SPIONs was applied in the model. Data for skin, muscles, intestine, blood adipose and testes were not available in mice.

the model compared with experimental data for the doses of 1020 and $2040 \mu \mathrm{g}$. The SPIONs concentration in vivo was $0.15 \mu \mathrm{g} / \mathrm{g}$ vs. $1.24 \mu \mathrm{g} / \mathrm{g}$ and $0.28 \mu \mathrm{g} / \mathrm{g}$ vs. $2.27 \mu \mathrm{g} / \mathrm{g}$ in the model, respectively.

Simulated concentrations were compared with observed SPIONs concentrations in mice taking into account the three doses. Organ concentrations have been normalized by the dose (organ concentrations/administered dose) and expressed as ng/g tissue/ $\mu$ g dose. As represented in Figure 5, mean simulated vs. observed SPION concentrations ( $\mathrm{ng} / \mathrm{g}$ tissue/ $\mu \mathrm{g}$ dose) were equal to 11.40 vs. 18.67 (ratio $=1.63$ ) in the spleen, 12.30 vs. 13.28 (ratio $=1.07)$ in the liver, 2.66 vs. 12.43 (ratio=4.66) in the heart, 7.57 vs. 11.41 (ratio =1.50) in the lung, 2.15 vs. 1.20 


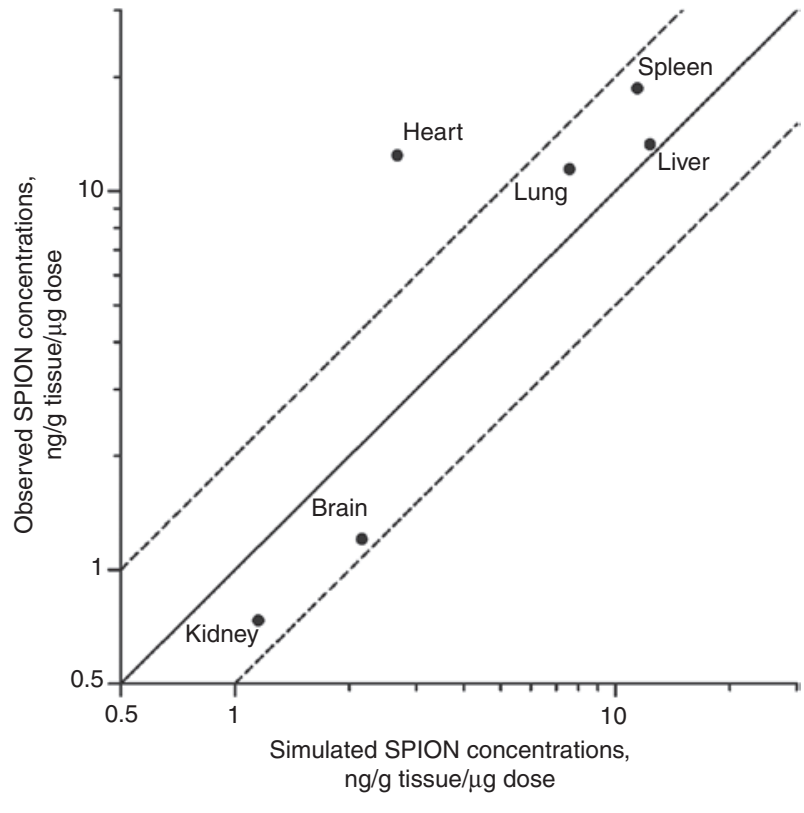

Figure 5: Scatter dot representing the SPIONs concentrations normalized considering the dose resulting from simulated and observed administration of SPIONs. The plotted value are the average SPIONs concentrations normalized considering the dose in mice treated with 510,1020 and $2040 \mu \mathrm{g} /$ mouse and the results are expressed as ng of SPIONs per gram of tissue/ $\mu \mathrm{g}$ of dose.

(ratio $=1.78$ ) in the brain and 1.15 vs. 0.73 (ratio=1.56) in the kidney.

\section{Human PBPK model}

The simulated pharmacokinetics of SPIONs is summarized in Figure 6. The distribution of SPIONs was characterized

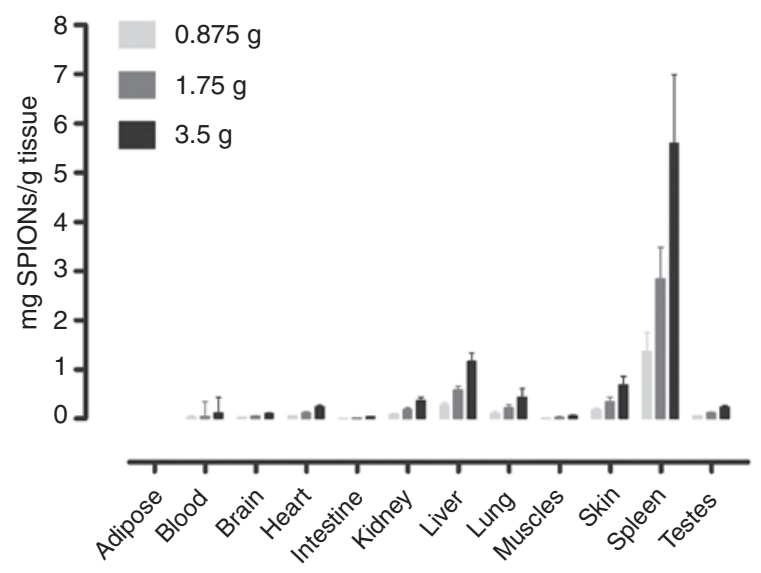

Figure 6: Simulated data accumulation of SPIONs in human. The dose administered was $0.875,1.75$ and $3.5 \mathrm{~g}$ of SPIONs per human and the results are expressed as milligram of SPIONs per gram of tissue. by high concentrations in the spleen, liver and skin, with accumulation of $5.59 \mathrm{mg}$ in the spleen, $1.16 \mathrm{mg}$ in the liver and $0.69 \mathrm{mg}$ in the skin.

\section{Discussion}

The aim of this study was to develop a PBPK model to predict the distribution and accumulation of NPs in vivo. Novel in vitro experimental data describing uptake of SPIONs in murine macrophage cell line and primary human monocyte-derived macrophages were integrated into this computational approach. NP penetration into tissues was modeled considering the diffusion of NPs through the capillary fenestration and the phagocytic activity mediated by macrophages (38).

Although several studies have identified SPIONs as a promising technology platform for various applications, there is a limited understanding of the pharmacokinetics and this represents a major obstacle for their translation. In this scenario, the construction of predictive PBPK models for humans can effectively contribute (i) to development of new tools that combine in vitro and ex vivo data providing a better understanding of NPs distribution in vivo; (ii) to the creation of a theoretical approach to risk assessment for NPs that reduces reliance on the use of animals; (iii) to accelerate studies to understand the correlation between NPs physical properties and distribution that can inform design of future nanomaterials.

Several properties such as size, surface chemistry and shape can influence the interactions between NPs and plasma proteins, cellular uptake and toxicity $(39,40)$. The biodistribution and pharmacokinetics of inorganic NPs has been investigated across different technology platforms. Three different surface-modified $45 \mathrm{~nm}$ silica NPs showed different half-life $\left(t_{1 / 2}\right)$ in vivo. PEG-coated silica NP (PEG-SiNP) remained in the circulation longer $\left(\mathrm{t}_{1 / 2}=180 \mathrm{~min}\right)$ than hydroxyl-coated silica NPs (OH-SiNP, $\mathrm{t}_{1 / 2}=80 \mathrm{~min}$ ) and carboxyl-coated silica NPs (COOH-SiNP, $\mathrm{t}_{1 / 2}=35 \mathrm{~min}$ ). Both OH-SiNP and COOH-SiNP accumulated in the liver $3 \mathrm{~h}$ after administration, while no signal of PEG-SiNP was detected in hepatic tissues up to $24 \mathrm{~h}$ (41). Similarly, gold-dendrimers of $5 \mathrm{~nm}$ with negative and neutral surface charge were described to accumulate mainly in the liver and spleen whereas positively-charged dendrimers showed accumulation predominantly in the kidney and blood (42). The impact of size on the distribution and elimination of PEG-coated gold NPs (PEG-AuNP) was investigated by Cho et al. (43) using small (4 or $13 \mathrm{~nm}$ ) and large (100 nm) NPs. The concentrations of small NPs 
remained high for $24 \mathrm{~h}$ in the blood whereas large NPs had faster clearance and elimination in $24 \mathrm{~h}$. These results are in accordance with previous studies, in which NPs with diameter below $10 \mathrm{~nm}$ were characterized by prolonged circulation time (44). Therefore, size and surface chemistry clearly influence pharmacokinetics and distribution.

The simulated pharmacokinetics of SPIONs in the mouse PBPK model presented here was in good accordance with the in vivo experimental data, with higher accumulation predicted in the spleen, lung and liver. Higher accumulation in the spleen, liver and lungs can be influenced by the protein corona that forms on the surface of NPs that mediates the interaction with cells and tissues and can enhance the uptake and accumulation of NPs in cells $(45,46)$. Therefore, the predicted distribution pattern is in accordance with previously published data describing higher accumulation in macrophage-rich organs such as spleen, liver and lung (47-50).

Murine macrophage cell line was used to determine the uptake of SPIONs as a surrogate for the RES system in vivo in mice. As the simulated tissue accumulation is in accordance with the in vivo experimental data in mice, the in vitro and in silico approaches were used to predict SPIONs distribution in humans. The PBPK model was used to provide a physiological and anatomical description of the mechanisms regulating NPs distribution in humans, integrating the quantification of SPIONs apparent uptake in human primary macrophages for the prediction of RES clearance in liver, spleen and lungs. This has the potential of representing a valuable tool to bridge investigation of NPs pharmacokinetics from pre-clinical species to humans, generating data across a wider range of materials with a better understanding of how NPs properties such as size, shape or even the possibility of protein corona formation can influence their distribution.

In mice, the main difference between the experimental and modeled data was found in kidney, in which the experimental accumulation is lower. Although our prediction were overall correct, there is a fundamental lack of understanding related to the mechanism involved in accumulation of SPIONs in certain tissues. Consequently, additional experimental investigations including other population of cell to further elucidate the mechanisms regulating SPIONs penetration in tissues are warranted. An additional limitation could be represented by potential ADME differences among species used in preclinical studies, where accumulation of SPIONs or other nanomaterials in specific organs might be substantially different and influenced by nanoparticle's properties (i.e. size, charge and chemical structure) as well as experimental conditions (51).
The accumulated NPs in cells or tissues can become toxic or disrupt normal cell chemistry, resulting in harmful side effects, as already described for many authors (43, 52-55). Therefore, the identification of NPs properties influencing distribution and accumulation is essential and may support the rational selection of technology platforms for specific biomedical applications. As there is no data available in the literature regarding the distribution and accumulation of several types of NPs in human, the theoretical approach to risk assessment may constitute a valuable approach.

The distribution of NPs in humans and animals is mediated by numerous physiological processes in several tissues and organs which substantially differ from the pharmacokinetics of traditional formulations. Consequently, the generation of PBPK models for NPs is challenging, due to incomplete understanding of the molecular mechanisms defining NPs distribution as well as a limited availability of experimental approaches to inform computational models. In this work, the results obtained from the human PBPK model described a similar distribution found in the murine PBPK, with a higher NPs accumulation in spleen and lung. As expected, the accumulation increased with higher NPs dose. These data should be interpreted in the context that many of the input data were the same for both models and the mouse model accurately predicted accumulation in five out of six tissues (considering a $\approx 0.5-2$ fold cut-off). With the discovery of novel, as well as a better characterization of the known mechanisms mediating SPIONs pharmacokinetics as more advanced in vitro assays and PBPK models are developed, the precision and accuracy of this computational approach is likely to increase.

\section{Conclusions}

In conclusion, the PBPK model developed showed accuracy in predicting the SPIONs accumulation in mice, especially in the liver, spleen and lung, when compared with experimental results. The results obtained from the human PBPK model described a similar distribution found in the murine PBPK, with a higher NPs accumulation in spleen and liver. Also, the in vitro approach using mice and human macrophages as an alternative to surrogate the RES in vivo, showed appropriate for the SPIONs accumulation prediction, since the similarity of obtained results.

The application of modeling techniques may have value in bridging between pre-clinical and human NPs pharmacokinetics. The integration of in vitro assays for ADME processes can improve PBPK approaches, allowing 
clarification of how NPs properties influence distribution patterns. Consequently, although there are current gaps in knowledge, PBPK models have the potential to support the design of NPs by providing a rational approach for the selection of materials to build up new nanostructured systems with optimal pharmacological properties, as well as, biocompatibility.

The development of this model can support the idea of using a theoretical approach to surrogate the pharmacokinetic of nanoparticles, using in vitro uptake assays to take into account the nanoparticle properties and physicochemical characteristics. Since the uptake study is used, other nanoparticles can be included in the model, avoiding or minimizing the use of animals. This model represents an initial platform to provide computational prediction of nanoparticle distribution and aims to promote future expansions of this approach, where additional variants such as other cell populations or nanoparticle types can be included.

Acknowledgments: This study was supported by doctoral fellowship from CAPES (Coordenação de Aperfeiçoamento de Pessoal de Nivel Superior), doctoral sandwich scholarship from Science Without Borders (Grant/Award Number: 'BEX 0378/13-5') (Ciências sem Fronteiras) and CPNq (Conselho Nacional de Desenvolvimento Científico e Tecnológico) and makes part of the doctoral activities of Adny Henrique Silva.

Conflict of interest statement: The authors state no conflict of interest. All authors have read the journal's Publication ethics and publication malpractice statement available at the journal's website and hereby confirm that they comply with all its parts applicable to the present scientific work.

\section{References}

1. Arbab AS, Bashaw LA, Miller BR, Jordan EK, Lewis BK, Kalish H, et al. Characterization of biophysical and metabolic properties of cells labeled with superparamagnetic iron oxide nanoparticles and transfection agent for cellular MR imaging. Radiology 2003;229:838-46.

2. Mahmoudi M, Sant S, Wang B, Laurent S, Sen T. Superparamagnetic iron oxide nanoparticles (SPIONs): development, surface modification and applications in chemotherapy. Adv Drug Deliv Rev 2001;63:24-46.

3. Chen R, Ratnikova TA, Stone MB, Lin S, Lard M, Huang G, et al. Differential uptake of carbon nanoparticles by plant and Mammalian cells. Small 2010;6:612-7.

4. Kolosnjaj-Tabi J, Wilhelm C, Clément 0, Gazeau F. Cell labeling with magnetic nanoparticles: opportunity for magnetic cell imaging and cell manipulation. J Nanobiotechnol 2013;11:1-19.
5. Gupta AK, Gupta M. Synthesis and surface engineering of iron oxide nanoparticles for biomedical applications. Biomaterials 2005;26:3995-4021.

6. Silva AC, Oliveira TR, Mamani JB, Malheiros SMF, Malavolta L, Pavon LF, et al. Application of hyperthermia induced by superparamagnetic iron oxide nanoparticles in glioma treatment. Int J Nanomedicine 2011;6:591-603.

7. Barhoumi L, Dewez D. Toxicity of superparamagnetic iron oxide nanoparticles on green alga Chlorella vulgaris. Biomed Res Int 2013;2013:1-11.

8. Park E-J, Choi D-H, Kim Y, Lee E-W, Song J, Cho M-H, et al. Magnetic iron oxide nanoparticles induce autophagy preceding apoptosis through mitochondrial damage and ER stress in RAW264.7 cells. Toxicol In Vitro 2014;28:1402-12.

9. Sarkar A, Sil PC. Iron oxide nanoparticles mediated cytotoxicity via PI3K/AKT pathway: role of quercetin. Food Chem Toxicol 2014;71:106-15.

10. Singh N, Jenkins GJS, Asadi R, Doak SH. Potential toxicity of superparamagnetic iron oxide nanoparticles (SPION). Nano Rev 2010;1:1-16.

11. Li M, Al-jamal KT, Kostarelos K, Reineke J. Physiologically based pharmacokinetic modeling of nanoparticles. ACS Nano 2010;4:6303-17.

12. Moss DM, Siccardi M. Optimizing nanomedicine pharmacokinetics using physiologically based pharmacokinetics modelling. $\mathrm{Br}$ J Pharmacol 2014;171:1-17.

13. Chazaud B. Macrophages: supportive cells for tissue repair and regeneration. Immunobiology 2014;219:172-8.

14. Mojica Pisciotti ML, Lima E, Vasquez Mansilla M, Tognoli VE, Troiani HE, Pasa AA, et al. In vitro and in vivo experiments with iron oxide nanoparticles functionalized with DEXTRAN or polyethylene glycol for medical applications: magnetic targeting. J Biomed Mater Res Part B Appl Biomater 2014;102:860-8.

15. Li S, Huang L. Pharmacokinetics and biodistribution of nanoparticles. Mol Pharm 2008;4:496-504.

16. Caliceti P, Veronese FM. Pharmacokinetic and biodistribution properties of poly(ethylene glycol)-protein conjugates. Adv Drug Deliv Rev 2003;55:1261-77.

17. Silva AH, Lima E, Mansilla MV, Zyesler RD, Trioani H, Pisciotti MLM, et al. Superparamagnetic iron-oxide nanoparticles mPEG350- and mPEG2000-coated: cell uptake and biocompatibility evaluation. Nanomedicine 2016;1:1-10.

18. Vargas JM, Zysler RD. Tailoring the size in colloidal iron oxide magnetic nanoparticles. Nanotechnology 2005;16:1474-6.

19. Silva AH, Filippin-Monteiro FB, Mattei B, Zanetti-Ramos BG, Creczynski-Pasa TB. In vitro biocompatibility of solid lipid nanoparticles. Sci Total Environ 2012;432:382-8.

20. Zysler RD, Lima Jr E, Mansilla MV, Troiani HE, Pisciotti MLM, Gurman P, et al. A new quantitative method to determine the uptake of SPIONs in animal tissue and its application to determine the quantity of nanoparticles in the liver and lung of Balb-c mice exposed to the SPIONs. J Biomed Nanotechnol 2013;9:142-5.

21. Riemer J, Hoepken HH, Czerwinska H, Robinson SR, Dringen R. Colorimetric ferrozine-based assay for the quantitation of iron in cultured cells. Anal Biochem 2004;331:370-5.

22. Sakoda A, Ishimori Y, Kawabe A, Kataoka T, Hanamoto K, Yamaoka K. Physiologically based pharmacokinetic modeling of inhaled radon to calculate absorbed doses in mice, rats, and humans. J Nucl Sci Technol 2010;47:731-8. 
23. Brown RP, Delp MD, Lindstedt SL, Rhomberg LR, Beliles RP. Physiological parameter values for physiologically based pharmacokinetic models. Toxicol Ind Health 1997;13:407-84.

24. Rodriguez M, Panda BB, Ficsor G. Testes weight reflect ethylnitrosourea induced histopathology in mice. Toxicol Lett 1983;17:77-80.

25. Zhang F, Tagen M, Throm S, Mallari J, Miller K, Guy RK, et al. Whole-body physiologically based pharmacokinetic model for nutlin-3a in mice after intravenous and oral administration. Drug Metab Dispos 2011;39:15-21.

26. Bachler G, von Goetz N, Hungerbühler K. A physiologically based pharmacokinetic model for ionic silver and silver nanoparticles. Int J Nanomed 2013;8:3365-82.

27. Utturkar A, Paul B, Akkiraju H, Bonor J, Dhurjati P, Nohe A. Development of physiologically based pharmacokinetic model (PBPK) of BMP2 in mice. Biol Syst 2013;2:1-15.

28. Bosgra S, van Eijkeren J, Bos P, Zeilmaker M, Slob W. An improved model to predict physiologically based model parameters and their inter-individual variability from anthropometry. Crit Rev Toxicol 2012;42:751-67.

29. Lankveld DPK, Oomen AG, Krystek P, Neigh A, Troost-de Jong A, Noorlander CW, et al. The kinetics of the tissue distribution of silver nanoparticles of different sizes. Biomaterials 2010;31:8350-61.

30. Bachler G, Hungerbühler K. A physiologically based pharmacokinetic model for ionic silver and silver nanoparticles. Int J Nanomedicine 2013;8:3365-82.

31. Davies LC, Jenkins SJ, Allen JE, Taylor PR. Tissue-resident macrophages. Nat Immunol 2013;14:986-95.

32. Moghimi SM, Hunter AC, Murray JC. Long-circulating and target-specific nanoparticles: theory to practice. Pharmacol Rev 2011;53:283-318.

33. Friedman SL, Rockey DC, McGuire RF, Maher JJ, Boyles JK, Yamasaki G. Isolated hepatic lipocytes and Kupffer cells from normal human liver: morphological and functional characteristics in primary culture. Hepatology 1992;15:234-43.

34. Ten Hagen TLM, Van Vianen W, Bakker-Woudenberg IA. Isolation and characterization of murine Kupffer cells and splenic macrophages. J Immunol Methods 1996;193:81-91.

35. Blackley S, Kou Z, Chen H, Quinn M, Rose RC, Schlesinger JJ, et al. Primary human splenic macrophages, but not T or B cells, are the principal target cells for dengue virus infection in vitro. J Virol 2007;81:13325-34.

36. Wang C, Yu X, Cao Q, Wang Y, Zheng G, Tan TK, et al. Characterization of murine macrophages from bone marrow, spleen and peritoneum. BMC Immunol 2013;14:1-6.

37. Holt PG, Degebrodt A, Venaille T, O’Leary C, Krska K, Flexman J, et al. Preparation of interstitial lung cells by enzymatic digestion of tissue slices: preliminary characterization by morphology and performance in functional assays. Immunology 1985;54:139-47.

38. Brandenberger C, Mühlfeld C, Ali Z, Lenz A-G, Schmid O, Parak WJ, et al. Quantitative evaluation of cellular uptake and trafficking of plain and polyethylene glycol-coated gold nanoparticles. Small 2010;6:1669-78.

39. Aggarwal P, Hall JB, McLeland CB, Dobrovolskaia MA, McNeil SE. Nanoparticle interaction with plasma proteins as it relates to particle biodistribution, biocompatibility and therapeutic efficacy. Adv Drug Deliv Rev 2009;61:428-37.

40. Luciani N, Gazeau F, Wilhelm C. Reactivity of the monocyte/macrophage system to superparamagnetic anionic nanoparticles. J Mater Chem 2009;19:6373.
41. He X, Nie H, Wang K, Tan W, Wu X, Zhang P. In vivo study of biodistribution and urinary excretion of surface-modified silica nanoparticles. Anal Chem 2008;80:9597-603.

42. Balogh L, Nigavekar SS, Nair BM, Lesniak W, Zhang C, Sung LY, et al. Significant effect of size on the in vivo biodistribution of gold composite nanodevices in mouse tumor models. Nanomedicine 2007;3:281-96.

43. Cho W-S, Cho M, Jeong J, Choi M, Han BS, Shin H-S, et al. Sizedependent tissue kinetics of PEG-coated gold nanoparticles. Toxicol Appl Pharmacol 2010;245:116-23.

44. Heather AE, Michael AM. Detection methods for the in vivo biodistribution of iron oxide and silica. 2nd ed. Nanotoxicology. Boca Raton, FL: CRC Press, 2014:177-200.

45. Lesniak A, Fenaroli F, Monopoli MP, Åberg C, Dawson KA, Salvati A. Effects of the presence or absence of a protein corona on silica nanoparticle uptake and impact on cells. ACS Nano 2012;6:5845-57.

46. Yan Y, Gause KT, Kamphuis MMJ, Ang C-S, O’Brien-Simpson NM, Lenzo JC, et al. Differential roles of the protein corona in the cellular uptake of nanoporous polymer particles by monocyte and macrophage cell lines. ACS Nano 2013;7:10960-70.

47. Chertok B, Cole AJ, David AE, Yang VC. Comparison of electron spin resonance spectroscopy and inductively-coupled plasma optical emission spectroscopy for biodistribution analysis of iron-oxide nanoparticles. Mol Pharm 2010;7:375-85.

48. Cole AJ, David AE, Wang J, Galbán CJ, Yang VC. Magnetic brain tumor targeting and biodistribution of long-circulating PEGmodified, cross-linked starch-coated iron oxide nanoparticles. Biomaterials 2011;32:6291-301.

49. Jain TK, Reddy MK, Morales MA, Leslie-pelecky DL, Labhasetwar V. Biodistribution, clearance, and biocompatibility of iron oxide magnetic nanoparticles in rats. Mol Pharm 2008;5:316-27.

50. Liu SY, Han Y, Yin LP, Long L, Liu R. Toxicology studies of a superparamagnetic iron oxide nanoparticle in vivo. Adv Mater Res 2008;47-50:1097-100.

51. Tietze R, Lyer S, Dürr S, Struffert T, Engelhorn T, Schwarz M, et al. Efficient drug-delivery using magnetic nanoparticles - biodistribution and therapeutic effects in tumour bearing rabbits. Nanomed Nanotech Biol Med 2013;9:961-71.

52. Wang J, Chen Y, Chen B, Ding J, Xia G, Gao C, et al. Pharmacokinetic parameters and tissue distribution of magnetic $\mathrm{Fe}_{3} \mathrm{O}_{4}$ nanoparticles in mice. Int J Nanomedicine 2010;5:861-6.

53. Zhu M-T, Feng W-Y, Wang Y, Wang B, Wang M, Ouyang H, et al. Particokinetics and extrapulmonary translocation of intratracheally instilled ferric oxide nanoparticles in rats and the potential health risk assessment. Toxicol Sci 2009;107:342-51.

54. Kumar R, Roy I, Ohulchanskky TY, Vathy LA, Bergey EJ, Sajjad $M$, et al. In vivo biodistribution and clearance studies using multimodal organically modified silica nanoparticles. ACS Nano 2010;4:699-708.

55. Cho W-S, Cho M, Jeong J, Choi M, Cho H-Y, Han BS, et al. Acute toxicity and pharmacokinetics of $13 \mathrm{~nm}$-sized PEG-coated gold nanoparticles. Toxicol Appl Pharmacol 2009;236:16-24.

Supplemental Material: The online version of this article (DOI: 10.1515/ejnm-2017-0001) offers supplementary material, available to authorized users. 


\section{Bionotes}

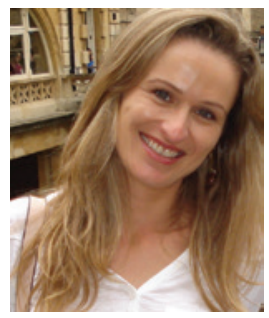

\section{Adny Henrique Silva}

Departamento de Ciências Farmacêuticas, Universidade Federal de Santa Catarina, P.O. Box 476, Florianópolis, SC, 88040-900, Brazil, Phone: +554837212212 ,

Fax: +554837219542 ,

adnyh@yahoo.com.br; adnyhe@gmail.com

Adny Henrique Silva obtained her PhD (2015) and her Master's degree (2011) in Pharmacy from the Federal University of Santa Catarina, Florianópolis, Brazil. She is at present, a postdoctoral researcher in Biochemistry department of the same University. Her $\mathrm{PhD}$ research was on "evaluation of nanoparticles toxicity using in vitro, in vivo, ex vivo and in silico models". During this period, she also had the opportunity to participate in other projects that were anticancer-related, including the gene modulation through siRNA delivery using nanocarriers. She is experienced in cell biology, biochemistry, toxicology and nanotechnology. She has been working for the past 7 years on the investigation of antitumor properties of new molecules and new strategies for cancer diagnosis as well as new strategies to study the toxicity of nanoparticles. She also had the opportunity of 10 months' collaborative research at the University of Liverpool, England.

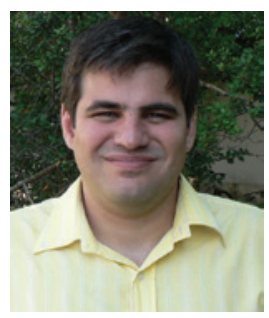

Enio Lima Jr

División of Resonancias Magnéticas, Centro Atómico Bariloche/CONICET, S. C. Bariloche 8400 , Argentina

Enio Lima Jr works in the Centro Atómico Bariloche/CONICET, Argentina, as an independent researcher. He graduated in Physics and with a PhD in Physics of condensed matter at the Universidade Federal de Santa Catarina, Brazil, he has been working for more than 10 years in the study of magnetic nanoparticles and their applications.

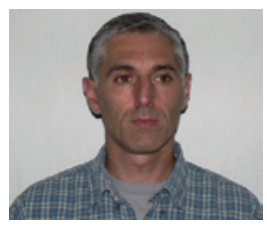

Marcelo Vasquez Mansilla

División of Resonancias Magnéticas, Centro Atómico Bariloche/CONICET, S. C. Bariloche 8400 , Argentina

Marcelo Vasquez Mansilla was born in Buenos Aires, Argentina in 1972. He received his Bachelor's degree in Physics from Universidad Nacional de Cuyo, Argentina in 1997 and his PhD in Physics in 2004. He worked for 2 years at Istituto di Struttura della Materia del CNR di Roma and since 2007 he has held a position as a researcher at the Magnetic Resonance Laboratory in Centro Atomico Bariloche, Argentina. His current research focus on magnetism of nanostructure materials like magnetic nanoparticles and thin films. He is also involved in applied research using nanoparticles in medicine as magnetic hiperthermia, drug delivery and sensors.

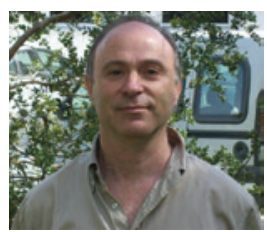

Roberto Daniel Zysler

División of Resonancias Magnéticas, Centro Atómico Bariloche/CONICET, S. C. Bariloche 8400 , Argentina

Roberto Daniel Zysler obtained his MS degree in Physics in 1985 and in 1992 he obtained his PhD in Physics at the Instituto Balseiro (Universidad Nacional de Cuyo, Argentina). In 1995 he started a permanent research position as a scientific researcher (CONICET, Argentina) at the Magnetism Group of the Centro Atómico Bariloche (at the Magnetic Resonance Laboratory), and from this time he has worked on magnetic nanoparticles properties and their applications in bio-medicine. He is a Professor at the Instituto Balseior and advisor of Master's degree thesis and PhD thesis for Physics candidates. He has over 150 scientific publication in international scientific journals and presented over 170 congress communication contributions at international scientific meetings.

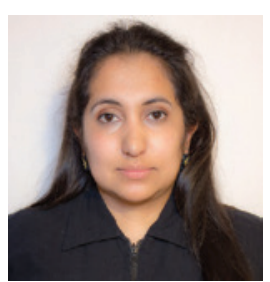

Mary Luz Mojica Pisciotti

División of Resonancias Magnéticas, Centro Atómico Bariloche/CONICET, S. C. Bariloche 8400 , Argentina

Mary Luz Mojica Pisciotti obtained her PhD in Physics and her MSc in Medical Physics from Balseiro Institute and National University of Cuyo in Argentina and her BSc in Physics from the National University of Colombia. She is currently a postdoctoral researcher at the National Atomic Energy Commission (CNEA) in Bariloche. Her research is focused on the use of magnetic nanoparticles (MNPs) in medicine, particularly in magnetic hyperthermia and in the application of these nanomaterials in contrast-enhanced magnetic resonance imaging (MRI). Mary has had the opportunity of working on various aspects of MNPs, including synthesis, magnetic and non-magnetic properties characterization and heating mechanisms. Additionally she has performed physical and biological experiments with iron oxide MNPs in different systems, including cellular and animal models. Her background has allowed her to explore the potential of working with interdisciplinary teams.

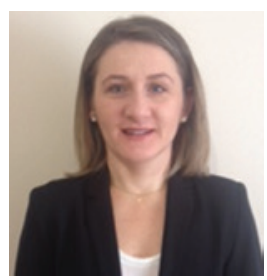

Claudriana Locatelli

Curso de Farmácia, Universidade do Oeste de Santa Catarina, Videira, SC, 89560-000, Brazil; and Universidade do Alto Vale do Rio do Peixe, Caçador, SC, 89500-000, Brazil

Claudriana Locatelli obtained her PhD in Pharmacy in 2009. She is currently a Professor of Biochemistry at the University of the West of Santa Catarina (Unoesc). She contributes as a Professor at Unoesc Postgraduate Program of Bioscience and Health, and to the Program of Science and Biotechnology. She has experience in antioxidants of natural products and cancer treatment. 


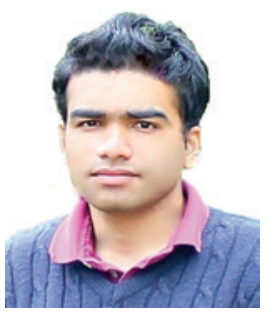

Rajith Kumar Reddy Rajoli

Molecular and Clinical Pharmacology, Institute of Translational Medicine, University of Liverpool, Liverpool, UK

Rajith Kumar Reddy Rajoli graduated in Pharmacy from the Birla Institute of Technology and Science, Pilani (BITS-Pilani), India in 2009. Later, he received his MSc in drug delivery from University College London, UK in 2011 and in 2017 he obtained a PhD in Pharmacology from the University of Liverpool, Liverpool, UK. Following his $\mathrm{PhD}$, he has been working as a research associate in the Molecular and Clinical Pharmacology department at the University of Liverpool. His current research focuses on informing the design of long-acting nanoparticles for intramuscular, subcutaneous and transdermal administration using physiologically based pharmacokinetic (PBPK) modeling.

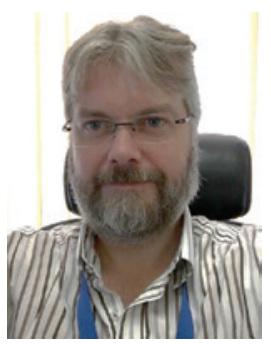

\section{Andrew Owen}

Molecular and Clinical Pharmacology, Institute of Translational Medicine, University of Liverpool, Liverpool, UK

Andrew Owen is Professor of Pharmacology at the University of Liverpool. He is Chair of the British Society for Nanomedicine, a Fellow of the Royal Society of Biology, and a Fellow of the British Pharmacological Society. His clinical and basic research focuses on understanding the mechanisms that underpin inter-patient variability in pharmacokinetics and pharmacodynamics, and to employ such knowledge to accelerate nanomedicine translation. His work is supported by the US Agency for International Development, the US National Institutes for Health, the UK Medical Research Council, the European Commission and the UK Engineering and Physical Sciences Research Council. He has published over 160 manuscripts, is co-inventor of patents relating to nanomedicines and a co-founder of Tandem Nano Ltd.

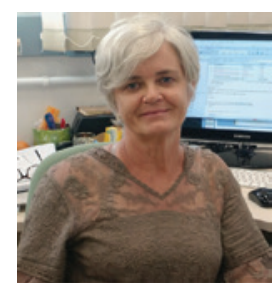

Tânia Beatriz Creczynski-Pasa

Departamento de Ciências Farmacêuticas, Universidade Federal de Santa Catarina, P.O. Box 476, Florianópolis, SC, 88040-900, Brazil

Tânia Beatriz Creczynski-Pasa graduated in Pharmacy from the Federal University of Rio Grande do Sul (1986), she obtained her Master's degree in Biological Chemistry at Federal University of Rio de Janeiro (1990) and her PhD in Biological Chemistry at Federal University of Rio de Janeiro (1994) with part of work at the University of Stuttgart (Germany). She is currently a full Professor at Federal University of Santa Catarina. She has experience in pharmacy and biochemistry, working mainly in the investigation of antitumor properties of new molecules and new strategies for cancer diagnosis. In parallel, she has been working in nanotechnology (especially in the development and analysis of siRNA carriers) and nanotoxicology. She has coordinated many projects, including the "Advanced diagnostic assessment for damage detection and determination of miRNAs as biomarkers for lung cancer".

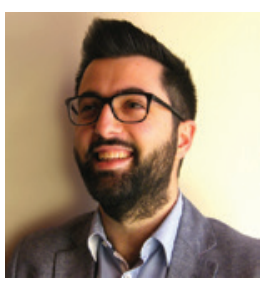

\section{Marco Siccardi}

Molecular and Clinical Pharmacology, Institute of Translational Medicine, University of Liverpool, Liverpool, UK

Marco Siccardi was appointed as a lecturer in Nanomedicine across the faculties of Health and Life Sciences and Science and Engineering at the University of Liverpool in 2012 and promoted to senior lecturer in Pharmacology in 2016. He has authored more than 80 peer reviewed publications, review manuscripts and book chapters. His research interests focus on the optimization of novel nanomedicine and traditional formulations for drug delivery based on experimental pharmacological data from in silico, in vitro and in vivo models, aiming to improve pharmacokinetics, efficacy and side effects. Additionally, he is interested in the clarification of the ADME processes involved in drug disposition and the identification of nanoformulation characteristics influencing drug exposure. 